\title{
Liquid biopsy enables real-time monitoring of molecular alterations in CRC
}

Liquid biopsy—-tracking circulating tumour DNA (ctDNA) in blood samples—can be used to genotype and monitor the molecular evolution of colorectal cancer (CRC), according to a new study. The approach can be used to monitor patients with CRC in real-time, enabling the identification of drug resistance and to track tumour dynamics.

"Although the presence of ctDNA in the blood of patients has been known for decades, only recent advances in genomics have allowed the detection and quantification of cancer-related molecular alterations with high specificity and sensitivity," explains author Alberto Bardelli. The researchers wanted to evaluate whether ctDNA analysis in patients with CRC had practical applications.

Using a variety of advanced sequencing techniques, the researchers assessed bloodbased molecular profiles of CRC tumours during a course of treatment with cetuximab and panitumumab (antibodies against epidermal growth factor receptor [EGFR]).
Detailed genomic profiles from ctDNA were obtained.

Liquid biopsy during the course of CRC treatment (including drug withdrawal and re-challenge) can be used for pharmacogenomic analysis and direct monitoring of treatment response, demonstrating that the CRC genome adapts dynamically to intermittent drug schedules. Alterations were observed in several genes (including KRAS and NRAS) upon analysis of ctDNA from patients with primary or acquired resistance to EGFR blockade.

Interestingly, mutated KRAS clones emerged during anti-EGFR antibody treatment and then declined upon withdrawal of this therapy, providing a molecular rationale for the efficacy of rechallenge with EGFR-targeted agents. Furthermore, drug-resistant cell populations and mutated clones were highly dynamic as the ctDNA profiles of individuals who benefitted from multiple rounds of treatment with anti-EGFR antibodies had pulsatile levels of mutant KRAS.

The authors believe that liquid biopsies will soon become part of routine clinical practice. "ctDNA can be used throughout the clinical management of patients with CRC to gather real-time updates on the molecular landscape of disease," notes Bardelli, adding that the mutational profiles between blood and tissue samples were highly concordant.

Further work is planned to use liquid biopsies systematically in clinical trials to closely monitor patients with CRC treated with targeted therapy and to measure residual disease after surgery.

Katrina Ray

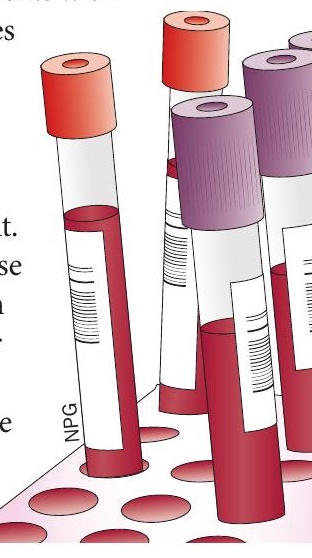

Original article Siravegna, G. et al. Clonal evolution and resistance to EGFR blockade in the blood of colorectal cancer patients. Nat. Med. doi:10.1038/nm.3870 